\title{
Geometrization of Monte-Carlo numerical analysis of an elliptic operator: strong approximation
}

\author{
Ana Bela Cruzeiro ${ }^{\mathrm{a}}$, Paul Malliavin ${ }^{\mathrm{b}}$, Anton Thalmaier $^{\mathrm{c}}$ \\ ${ }^{a}$ Grupo de Física-Matemática UL and Dep. Matemática IST, Av. Rovisco Pais, 1049-001 Lisboa, Portugal \\ b 10, rue Saint Louis en l'Isle, 75004 Paris, France \\ c Département de mathématiques, Université de Poitiers, téléport 2, BP 30179, 86962 Futuroscope Chasseneuil, France
}

Received 16 December 2003; accepted 12 January 2004

Presented by Paul Malliavin

\begin{abstract}
A one-step scheme is constructed, which, as the Milstein scheme, has the strong approximation property of order 1; in contrast to the Milstein scheme, our scheme does not involve the simulation of iterated Itô integrals of second order. To cite this article: A.B. Cruzeiro et al., C. R. Acad. Sci. Paris, Ser. I 338 (2004).

(C) 2004 Published by Elsevier SAS on behalf of Académie des sciences.
\end{abstract}

\section{Résumé}

Monte-Carlo géométrique pour un opérateur elliptique : approximation numérique forte. On propose un schéma à un pas, qui, comme le schéma de Milstein, possède la propriété d'approximation forte à l'ordre 1; contrairement au schéma de Milstein, notre schéma ne nécessite pas la simulation d'intégrales itérées de Itô du second degré. Pour citer cet article : A.B. Cruzeiro et al., C. R. Acad. Sci. Paris, Ser. I 338 (2004).

(C) 2004 Published by Elsevier SAS on behalf of Académie des sciences.

\section{Introduction}

Numerical integration of SDE consists, an SDE being fixed, in producing a discretization scheme leading to a pathwise approximation of its solution. In this work we discuss the related problem of how to establish a Monte-Carlo simulation to a given real strictly elliptic second order operator $\mathcal{L}$, which leads to good numerical approximations of the fundamental solution to the corresponding heat operator.

Of course many SDE can be associated to $\mathcal{L}$, each choice corresponding to a parametrization by the Wiener space of the Stroock-Varadhan solution of the martingale problem associated to $\mathcal{L}$. For applications to finance all these parametrizations are equivalent. We shall prove that there exists an optimal parametrization for which the one-step Milstein scheme does not involve the computation of iterated stochastic integrals of second order. For our

E-mail addresses: abcruz@math.ist.utl.pt (A.B. Cruzeiro), sli@ccr.jussieu.fr (P. Malliavin), thalmaier@math.univ-poitiers.fr (A. Thalmaier). 
search of an optimal parametrization we have to describe the possible parametrizations of the diffusion associated to $\mathcal{L}$; it is sufficient to solve this problem for the Euclidean Laplacian on $\mathbb{R}^{d}$; in this case it is equivalent to replace the standard Brownian motion $W$ on $\mathbb{R}^{d}$ by an orthogonal transform $\widetilde{W}$ with an Itô differential of the type

$$
\mathrm{d} \widetilde{W}_{k}=\sum_{j=1}^{d}\left[\Omega_{W}(t)\right]_{j}^{k} \mathrm{~d} W_{j}(t),
$$

where $t \mapsto \Omega_{W}(t)$ is an adapted process taking values in the group $\mathrm{O}(d)$ of orthogonal matrices. We denote by $\mathrm{O}(\mathcal{W})$ the family of all such orthogonal transforms which is isomorphic to $\mathbb{P}(\mathrm{O}(d))$, the path space on $\mathrm{O}(d)$. Pointwise multiplication defines a group structure on $\mathbb{P}(\mathrm{O}(d)) \simeq \mathrm{O}(\mathcal{W})$.

Given on $\mathbb{R}^{d}$ the data of $d+1$ smooth vector fields $A_{0}, A_{1}, \ldots, A_{d}$, we consider the Itô SDE

$$
\mathrm{d} \xi_{W}(t)=A_{0}\left(\xi_{W}(t)\right) \mathrm{d} t+\sum_{k=1}^{d} A_{k}\left(\xi_{W}(t)\right) \mathrm{d} W_{k}(t), \quad \xi_{W}(0)=\xi_{0} .
$$

Throughout this Note we assume ellipticity, that is, for any $\xi \in \mathbb{R}^{d}$ the vectors $A_{1}(\xi), \ldots, A_{d}(\xi)$ constitute a basis of $\mathbb{R}^{d}$; the components of a vector field $U$ in this basis are denoted $\left\langle U, A_{k}\right\rangle_{\xi}$ which gives the decomposition $U(\xi)=\sum_{k=1}^{d}\left\langle U, A_{k}\right\rangle_{\xi} A_{k}(\xi)$. By change of parametrization we mean the substitution of $W$ by $\widetilde{W}$ in (2); we then get an Itô process in $\widetilde{W}$. This change of parametrization does not change the infinitesimal generator associated to (2) which has the form $\mathcal{L}=\frac{1}{2} \sum_{k, \alpha, \beta} A_{k}^{\alpha} A_{k}^{\beta} D_{\alpha} D_{\beta}+\sum_{\alpha} A_{0}^{\alpha} D_{\alpha}$ where $D_{\alpha}=\partial / \partial \xi^{\alpha}$. The group O(W) operates on the set of elliptic SDE on $\mathbb{R}^{d}$ and the orbits of this action are classified by the corresponding elliptic operators $\mathcal{L}$.

\section{Definition of the scheme $\&$}

Denote by $t_{\varepsilon}:=\varepsilon \times$ integer part of $t / \varepsilon$; we define our scheme by

$$
\begin{aligned}
& Z_{W^{\varepsilon}}(t)-Z_{W^{\varepsilon}}\left(t_{\varepsilon}\right)=A_{0}\left(Z_{W^{\varepsilon}}\left(t_{\varepsilon}\right)\right)\left(t-t_{\varepsilon}\right)+\sum_{k} A_{k}\left(Z_{W^{\varepsilon}}\left(t_{\varepsilon}\right)\right)\left(W_{k}(t)-W_{k}\left(t_{\varepsilon}\right)\right) \\
& +\frac{1}{2} \sum_{k, s}\left(\partial_{A_{k}} A_{s}\right)\left(Z_{W^{\varepsilon}}\left(t_{\varepsilon}\right)\right)\left\{\left(W_{k}(t)-W_{k}\left(t_{\varepsilon}\right)\right)\left(W_{s}(t)-W_{s}\left(t_{\varepsilon}\right)\right)-\varepsilon \eta_{k}^{s}\right\} \\
& +\frac{1}{2} \sum_{k, s, i} A_{i}\left(Z_{W^{\varepsilon}}\left(t_{\varepsilon}\right)\right)\left\langle\left[A_{s}, A_{i}\right], A_{k}\right\rangle_{Z_{W^{\varepsilon}}\left(t_{\varepsilon}\right)}\left\{\left(W_{k}(t)-W_{k}\left(t_{\varepsilon}\right)\right)\left(W_{s}(t)-W_{s}\left(t_{\varepsilon}\right)\right)-\varepsilon \eta_{k}^{s}\right\},
\end{aligned}
$$

where $W$ is standard Brownian motion on $\mathbb{R}^{d}$, and $\eta_{k}^{s}$ the Kronecker symbol defined by $\eta_{k}^{s}=1$ if $k=s$ and zero otherwise. Denote by $\mathbb{P}\left(\mathbb{R}^{d}\right)$ the path space on $\mathbb{R}^{d}$, that is the Banach space of continuous maps from $[0, T]$ into $\mathbb{R}^{d}$, endowed with the sup norm: $\left\|p_{1}-p_{2}\right\|_{\infty}=\sup _{t \in[0, T]}\left|p_{1}(t)-p_{2}(t)\right|_{\mathbb{R}^{d}}$. Fixing $\xi_{0} \in \mathbb{R}^{d}$, let $\mathbb{P}_{\xi_{0}}\left(\mathbb{R}^{d}\right)$ be the subspace of paths starting from $\xi_{0}$. Given Borel measures $\rho_{1}, \rho_{2}$ on $\mathbb{P}\left(\mathbb{R}^{d}\right)$, denote by $\mathcal{M}\left(\rho_{1}, \rho_{2}\right)$ the set of measurable maps $\Psi: \mathbb{P}\left(\mathbb{R}^{d}\right) \rightarrow \mathbb{P}\left(\mathbb{R}^{d}\right)$ such that $\Psi_{*} \rho_{1}=\rho_{2}$; the Monge transport norm (see $[8,5]$ ) is defined as

$$
d_{\mathcal{M}}\left(\rho_{1}, \rho_{2}\right):=\left[\inf _{\Psi \in \mathcal{M}\left(\rho_{1}, \rho_{2}\right)} \int\|\Psi(p)\|_{\infty}^{2} \rho_{1}(\mathrm{~d} p)\right]^{1 / 2} .
$$

Theorem 2.1. Assume ellipticity and assume the vector fields $A_{k}$ along with their first three derivatives to be bounded; fix $\xi_{0} \in \mathbb{R}^{d}$ and let $\rho_{\mathcal{L}}$ be the measure on $P_{\xi_{0}}\left(\mathbb{R}^{d}\right)$ defined by the solution of the Stroock-Varadhan martingale problem [7] for the elliptic operator $\mathcal{L} ;$ let $\rho$ \& be the measure obtained by the scheme $\&$ with initial value $Z_{W^{\varepsilon}}(0)=\xi_{0}$. Then

$$
\limsup _{\varepsilon \rightarrow 0} \frac{1}{\varepsilon} d_{\mathcal{M}}\left(\rho_{\mathcal{L}}, \rho_{\S}\right)=c<\infty .
$$


Remark. The proof of Theorem 2.1 will provide an explicit transport functional $\Psi_{0}$ which puts the statement in a constructive setting; the constant $c$ is effective.

\section{The Milstein scheme}

The Milstein scheme for SDE (2) (cf., for instance, [6], formula (0.23) or [2], p. 345; see also [3]) is based on the following stochastic Taylor expansion of $A_{k}$ along the diffusion trajectory: $A_{k}\left(\xi_{W}(t)\right)=A_{k}\left(\xi_{W}\left(t_{\varepsilon}\right)\right)+$ $\sum_{j}\left(\partial_{A_{j}} A_{k}\right)\left(\xi_{W}\left(t_{\varepsilon}\right)\right)\left(W_{j}(t)-W_{j}\left(t_{\varepsilon}\right)\right)+\mathrm{O}(\varepsilon)$, which leads to

$$
\begin{aligned}
\xi_{W^{\varepsilon}}(t)-\xi_{W^{\varepsilon}}\left(t_{\varepsilon}\right)= & \sum_{k} A_{k}\left(\xi_{W^{\varepsilon}}\left(t_{\varepsilon}\right)\right)\left(W_{k}(t)-W_{k}\left(t_{\varepsilon}\right)\right)+\left(t-t_{\varepsilon}\right) A_{0}\left(\xi_{W^{\varepsilon}}\left(t_{\varepsilon}\right)\right) \\
& +\sum_{i, k}\left(\partial_{A_{i}} A_{k}\right)\left(\xi_{W^{\varepsilon}}\left(t_{\varepsilon}\right)\right) \int_{t_{\varepsilon}}^{t}\left(W_{i}(s)-W_{i}\left(t_{\varepsilon}\right)\right) \mathrm{d} W_{k}(s) ;
\end{aligned}
$$

the computation of $\int_{t_{\varepsilon}}^{t}\left(W_{i}(s)-W_{i}\left(t_{\varepsilon}\right)\right) \mathrm{d} W_{k}(s)$ gives the Milstein scheme

$$
\begin{aligned}
\xi_{W^{\varepsilon}}(t)-\xi_{W^{\varepsilon}}\left(t_{\varepsilon}\right)= & \sum_{k} A_{k}\left(\xi_{W^{\varepsilon}}\left(t_{\varepsilon}\right)\right)\left(W_{k}(t)-W_{k}\left(t_{\varepsilon}\right)\right)+\left(t-t_{\varepsilon}\right) A_{0}\left(\xi_{W^{\varepsilon}}\left(t_{\varepsilon}\right)\right) \\
& +\frac{1}{2} \sum_{i, k}\left(\partial_{A_{i}} A_{k}\right)\left(\xi_{W^{\varepsilon}}\left(t_{\varepsilon}\right)\right)\left(\left(W_{i}(t)-W_{i}\left(t_{\varepsilon}\right)\right)\left(W_{k}(t)-W_{k}\left(t_{\varepsilon}\right)\right)-\varepsilon \eta_{k}^{i}\right)+R,
\end{aligned}
$$

where $R=\sum_{i<k}\left[A_{i}, A_{k}\right]\left(\xi_{W^{\varepsilon}}\left(t_{\varepsilon}\right)\right) \int_{t_{\varepsilon}}^{t}\left(W_{i}(s)-W_{i}\left(t_{\varepsilon}\right)\right) \mathrm{d} W_{k}(s)-\left(W_{k}(s)-W_{k}\left(t_{\varepsilon}\right)\right) \mathrm{d} W_{i}(s)$.

It is well known that the Milstein scheme has the following strong approximation property:

$$
\mathbb{E}\left[\sup _{t \in[0,1]}\left\|\xi_{W}(t)-\xi_{W^{\varepsilon}}(t)\right\|^{2}\right]=\mathrm{O}\left(\varepsilon^{2}\right) .
$$

The numerical difficulty related to the Milstein scheme is how to achieve a fast simulation of $R$. The purpose of this work is to show that by a change of parametrization this simulation can be avoided.

\section{Horizontal parametrization}

Given $d$ independent vector fields $A_{1}, \ldots, A_{d}$ on $\mathbb{R}^{d}$, we take the vectors $A_{1}(\xi), \ldots, A_{d}(\xi)$ as basis at the point $\xi$; the functions $\beta_{k, s}^{i}$, called structural functions, are defined (see [1]) by:

$$
\beta_{k, \ell}^{i}(\xi)=\left\langle\left[A_{k}, A_{\ell}\right], A_{i}\right\rangle_{\xi}, \quad\left[A_{k}, A_{\ell}\right](\xi)=\sum_{i} \beta_{k, \ell}^{i}(\xi) A_{i}(\xi)
$$

The structural functions are antisymmetric with respect to the two lower indices. Consider the connection functions, defined from the structural functions by

$$
\Gamma_{k, s}^{i}=\frac{1}{2}\left(\beta_{k, s}^{i}-\beta_{s, i}^{k}+\beta_{i, k}^{s}\right)
$$

Let $\Gamma_{k}$ be the $d \times d$ matrix obtained by fixing the index $k$ in the three indices functions $\Gamma_{k, *}^{*}$. Then, by means of the antisymmetry of $\beta_{*, *}^{*}$ in the two lower indices, $\Gamma_{k}$ is an antisymmetric matrix:

$$
2\left(\Gamma_{k, s}^{i}+\Gamma_{k, i}^{s}\right)=\beta_{k, s}^{i}-\beta_{s, i}^{k}+\beta_{i, k}^{s}+\beta_{k, i}^{s}-\beta_{i, s}^{k}+\beta_{s, k}^{i}=0 .
$$

The matrix $\Gamma_{k}$ operates on the coordinate vectors of the basis $A_{s}(\xi)$ via $\Gamma_{k}\left(A_{s}\right)=\sum_{i} \Gamma_{k, s}^{i} A_{i}$. This gives $\Gamma_{k}\left(A_{s}\right)-$ $\Gamma_{s}\left(A_{k}\right)=\left[A_{k}, A_{s}\right]$ : the $i$ th component of the 1.h.s. is $\frac{1}{2}\left(\beta_{k, s}^{i}-\beta_{s, i}^{k}+\beta_{i, k}^{s}-\beta_{s, k}^{i}+\beta_{k, i}^{s}-\beta_{i, s}^{k}\right)=\beta_{k, s}^{i}$. Let $\mathbb{M}=$ $\mathbb{R}^{d} \times \mathcal{E}_{d}$ where $\mathcal{E}_{d}$ is the vector space of $d \times d$ matrices. Define on $\mathbb{M}$ vector fields $\tilde{A}_{k}, k=1, \ldots, d$, as follows: 


$$
\tilde{A}_{k}(\xi, e)=\left(\sum_{\ell} e_{k}^{\ell} A_{\ell}(\xi), \mathcal{N}_{k}(\xi, e)\right), \quad\left[\mathcal{N}_{k}\right]_{r}^{s}(\xi, e)=-\sum_{\ell, \ell^{\prime}} e_{k}^{\ell} e_{r}^{\ell^{\prime}} \Gamma_{\ell, \ell^{\prime}}^{s}(\xi), \quad \xi \in \mathbb{R}^{d}, e \in \mathcal{E}_{d} .
$$

Denoting for a vector $Z$ on $\mathbb{M}$ by $Z^{H}$ its projection on $\mathbb{R}^{d}$, we have:

Proposition 4.1. The vector fields $\tilde{A}_{k}$ satisfy the relation $\left[\tilde{A}_{k}, \tilde{A}_{s}\right]^{\mathrm{H}}=0$.

Proof. The horizontal component $\left[\partial_{\tilde{A}_{k}} \tilde{A}_{s}\right]^{\mathrm{H}}$ is given by

$$
\begin{aligned}
{\left[\partial_{\tilde{A}_{k}} \tilde{A}_{s}\right]^{\mathrm{H}} } & =\sum_{i} \tilde{A}_{k}^{i} \partial_{i} \tilde{A}_{s}^{\mathrm{H}}+\sum_{q}\left(\sum_{\alpha, \beta}\left[\mathcal{N}_{k}\right]_{\beta}^{\alpha} \partial_{\left(\begin{array}{c}
\alpha \\
\beta
\end{array}\right)} e_{s}^{q}\right) A_{q} \\
& =\sum_{i}\left(\sum_{\ell} e_{k}^{\ell} A_{\ell}^{i}\right)\left(\sum_{\ell^{\prime}} e_{s}^{\ell^{\prime}} \partial_{i} A_{\ell^{\prime}}\right)-\sum_{\alpha, \beta}\left(\sum_{\ell, \ell^{\prime}} e_{k}^{\ell} e_{\beta}^{\ell^{\prime}} \Gamma_{\ell, \ell^{\prime}}^{\alpha}\right)\left(\sum_{q} \eta_{\alpha}^{q} \eta_{\beta}^{s} A_{q}\right) \\
& =\sum_{i}\left(\sum_{\ell} e_{k}^{\ell} A_{\ell}^{i}\right)\left(\sum_{\ell^{\prime}} e_{s}^{\ell^{\prime}} \partial_{i} A_{\ell^{\prime}}\right)-\sum_{\alpha, \ell, \ell^{\prime}} e_{k}^{\ell} e_{s}^{\ell^{\prime}} \Gamma_{\ell, \ell^{\prime}}^{\alpha} A_{\alpha},
\end{aligned}
$$

using the fact that $\partial_{\left(\begin{array}{c}\alpha \\ \beta\end{array}\right)} e_{s}^{q}=\eta_{\alpha}^{q} \eta_{\beta}^{s}$. We finally get $\left[\partial_{\tilde{A}_{k}} \tilde{A}_{s}\right]^{\mathrm{H}}=\sum_{\ell, \ell^{\prime}} e_{k}^{\ell} e_{s}^{\ell^{\prime}}\left[\partial_{A_{\ell}} A_{\ell^{\prime}}-\sum_{\alpha} \Gamma_{\ell, \ell^{\prime}}^{\alpha} A_{\alpha}\right]$. Therefore the horizontal component of the commutator is

$$
\left[\tilde{A}_{k}, \tilde{A}_{s}\right]^{\mathrm{H}}=\sum_{\ell, \ell^{\prime}} e_{k}^{\ell} e_{s}^{\ell^{\prime}}\left[A_{\ell}, A_{\ell^{\prime}}\right]-\sum_{\alpha, \ell, \ell^{\prime}} e_{k}^{\ell} e_{s}^{\ell^{\prime}}\left(\Gamma_{\ell, \ell^{\prime}}^{\alpha}-\Gamma_{\ell^{\prime}, \ell}^{\alpha}\right) A_{\alpha}
$$

which vanishes since $\Gamma_{\ell}\left(A_{\ell^{\prime}}\right)-\Gamma_{\ell^{\prime}}\left(A_{\ell}\right)=\left[A_{\ell}, A_{\ell^{\prime}}\right]$.

Denote by $e^{\mathrm{T}}$ the transposed of the matrix $e$ and let $\tilde{A}_{0}(\xi, e)=\left(A_{0}(\xi),-\frac{1}{2} e J\right), J:=\sum_{k=1}^{d} \mathcal{N}_{k}^{\mathrm{T}} \mathcal{N}_{k}$. Consider the following Itô SDE on the vector space $\mathbb{M}$ :

$$
\mathrm{d} m_{W}=\sum_{k} \tilde{A}_{k}\left(m_{W}\right) \mathrm{d} W_{k}+\tilde{A}_{0}\left(m_{W}\right) \mathrm{d} t, \quad m_{W}(0)=\left(\xi_{0}, \text { Id }\right) .
$$

Proposition 4.2. Denote $m_{W}(t)=\left(\tilde{\xi}_{W}(t), e_{W}(t)\right)$, then $e_{W}(t)$ is an orthogonal matrix for $t \geqslant 0$, and

$$
f\left(\tilde{\xi}_{W}(t)\right)-\int_{0}^{t}(\mathcal{L} f)\left(\tilde{\xi}_{W}(s)\right) \mathrm{d} s \text { is a local martingale, for any } f \in C^{2}\left(\mathbb{R}^{d}\right) .
$$

Proof. We compute the stochastic differential of $e^{\mathrm{T}} e$ :

$$
\begin{aligned}
\mathrm{d}\left[e^{\mathrm{T}} e\right]_{\ell}^{\ell^{\prime}}=\sum_{k} d\left(e_{k}^{\ell} e_{k}^{\ell^{\prime}}\right)= & -\sum_{m, k, p}\left(\sum_{u} e_{k}^{\ell} e_{m}^{p} e_{k}^{u} \Gamma_{p, u}^{\ell^{\prime}}+\sum_{v} e_{k}^{\ell^{\prime}} e_{m}^{p} e_{k}^{v} \Gamma_{p, v}^{\ell}\right) \mathrm{d} W_{m} \\
& +\sum_{k}\left(e_{k}^{\ell}\left(\tilde{A}_{0}\right)_{k}^{\ell^{\prime}}+e_{k}^{\ell}\left(\tilde{A}_{0}\right)_{k}^{\ell^{\prime}}+\sum_{m, p, q, p^{\prime}, q^{\prime}} e_{m}^{p} e_{k}^{p^{\prime}} \Gamma_{p, p^{\prime}}^{\ell^{\prime}} e_{m}^{q} e_{k}^{q^{\prime}} \Gamma_{q, q^{\prime}}^{\ell}\right) \mathrm{d} t,
\end{aligned}
$$

where the last term of the drift comes from the Itô contraction $\sum_{k} \mathrm{~d} e_{k}^{\ell} * \mathrm{~d} e_{k}^{\ell^{\prime}}=\sum_{k}\left[\mathcal{N}_{k}^{\mathrm{T}} \mathcal{N}_{k}\right]_{\ell}^{\ell^{\prime}} \mathrm{d} t=J_{\ell}^{\ell^{\prime}} \mathrm{d} t$. The first two terms of the drift are computed by using the definition of $\tilde{A}_{0}: \sum_{k}\left[e_{k}^{\ell}\left(\tilde{A}_{0}\right)_{k}^{\ell^{\prime}}+e_{k}^{\ell^{\prime}}\left(\tilde{A}_{0}\right)_{k}^{\ell}\right]=-\frac{1}{2}\left[\left(e^{\mathrm{T}} e J\right)_{\ell}^{\ell^{\prime}}+\right.$ $\left(e^{\mathrm{T}} e J\right)_{\ell^{\prime}}^{\ell}$. Write $e^{\mathrm{T}} e=\mathrm{Id}+\sigma$, then the drift takes the form $-(\sigma J+J \sigma) / 2$. We compute the coefficient of $\mathrm{d} W_{m}$ : 


$$
-\sum_{k, p}\left(\sum_{u} e_{k}^{\ell} e_{m}^{p} e_{k}^{u} \Gamma_{p, u}^{\ell^{\prime}}+\sum_{v} e_{k}^{\ell^{\prime}} e_{m}^{p} e_{k}^{v} \Gamma_{p, v}^{\ell}\right)=-\sum_{p} e_{m}^{p}\left(\sum_{u}\left[e^{\mathrm{T}} e\right]_{\ell}^{u} \Gamma_{p, u}^{\ell^{\prime}}+\sum_{v}\left[e^{\mathrm{T}} e\right]_{\ell^{\prime}}^{v} \Gamma_{p, v}^{\ell}\right) .
$$

Using the antisymmetry $\Gamma_{p, \ell}^{\ell^{\prime}}=-\Gamma_{p, \ell^{\prime}}^{\ell}$ we obtain

$$
\mathrm{d} \sigma_{\ell^{\prime}}^{\ell}=-\sum_{m} \mathrm{~d} W_{m} \sum_{p} e_{m}^{p}\left(\sum_{u} \sigma_{\ell}^{u} \Gamma_{p, u}^{\ell^{\prime}}+\sum_{v} \sigma_{\ell^{\prime}}^{v} \Gamma_{p, v}^{\ell}\right)-\frac{1}{2}[\sigma J+J \sigma]_{\ell^{\prime}}^{\ell} \mathrm{d} t .
$$

Eq. (10), together with Eq. (8), gives an SDE with local Lipschitz coefficients for the triple $(\tilde{\xi}, e, \sigma)$; by uniqueness of the solution, as $\sigma(0)=0$, we deduce $\sigma(t)=0$ for all $t \geqslant 0$.

In terms of the new $\mathbb{R}^{d}$-valued Brownian motion $\widetilde{W}$ defined by $\mathrm{d} \widetilde{W}_{k}(t):=\sum_{\ell}\left[e_{W}(t)\right]_{\ell}^{k} \mathrm{~d} W_{\ell}$, we have

$$
\mathrm{d} \tilde{\xi}_{W}=\sum_{k} A_{k}\left(\tilde{\xi}_{W}(t)\right) \mathrm{d} \tilde{W}_{k}(t)+A_{0}\left(\tilde{\xi}_{W}(t)\right) \mathrm{d} t .
$$

\section{Reconstruction of the scheme $\&$}

We want to prove that our scheme $\delta$ is essentially the projection of the Milstein scheme $\left(\tilde{\xi}_{W^{\varepsilon}}, e_{W^{\varepsilon}}\right)$ for the solution $m_{W}=\left(\tilde{\xi}_{W}, e_{W}\right)$ of the SDE (8). In order to write the first component $\tilde{\xi}_{W^{\varepsilon}}$ we have to compute the horizontal part of $\partial_{\tilde{A}_{k}} \tilde{A}_{j}$, which has been done in the proof to Proposition 4.1: we get

$$
\begin{aligned}
& \tilde{\xi}_{W^{\varepsilon}}(t)-\tilde{\xi}_{W^{\varepsilon}}\left(t_{\varepsilon}\right) \\
& =A_{0}\left(\tilde{\xi}_{W^{\varepsilon}}\left(t_{\varepsilon}\right)\right)\left(t-t_{\varepsilon}\right)+\sum_{k, \ell}\left[e_{W^{\varepsilon}}\left(t_{\varepsilon}\right)\right]_{k}^{\ell} A_{\ell}\left(\tilde{\xi}_{W^{\varepsilon}}\left(t_{\varepsilon}\right)\right) \Delta\left(W_{k}\right) \\
& \quad+\frac{1}{2} \sum_{k, j}\left\{\sum_{\ell, \ell^{\prime}}\left[e_{W^{\varepsilon}}\left(t_{\varepsilon}\right)\right]_{k}^{\ell}\left[e_{W^{\varepsilon}}\left(t_{\varepsilon}\right)\right]_{j}^{\ell^{\prime}}\left(\partial_{A_{\ell}} A_{\ell^{\prime}}-\sum_{i} \Gamma_{\ell, \ell^{\prime}}^{i} A_{i}\right)\left(\tilde{\xi}_{W^{\varepsilon}}\left(t_{\varepsilon}\right)\right)\right\}\left(\Delta\left(W_{k}\right) \Delta\left(W_{j}\right)-\varepsilon \eta_{k}^{j}\right),
\end{aligned}
$$

where $\Delta\left(W_{k}\right)=W_{k}(t)-W_{k}\left(t_{\varepsilon}\right)$. By (5)

$$
\mathbb{E}\left[\sup _{t \in[0,1]}\left\|e_{W}(t)-e_{W^{\varepsilon}}(t)\right\|^{2}\right] \leqslant c \varepsilon^{2}, \quad \mathbb{E}\left[\sup _{t \in[0,1]}\left\|\tilde{\xi}_{W}(t)-\tilde{\xi}_{W^{\varepsilon}}(t)\right\|^{2}\right] \leqslant c \varepsilon^{2} .
$$

Consider the new process $\xi_{W}^{\sharp}$ defined by

$$
\begin{aligned}
& \xi_{W}^{\sharp}(t)-\xi_{W}^{\sharp}\left(t_{\varepsilon}\right)=A_{0}\left(\xi_{W}^{\sharp}\left(t_{\varepsilon}\right)\right)\left(t-t_{\varepsilon}\right)+\sum_{k, \ell}\left[e_{W}\left(t_{\varepsilon}\right)\right]_{k}^{\ell} A_{\ell}\left(\xi_{W}^{\sharp}\left(t_{\varepsilon}\right)\right) \Delta\left(W_{k}\right) \\
& \quad+\frac{1}{2} \sum_{k, j}\left\{\sum_{\ell, \ell^{\prime}}\left[e_{W}\left(t_{\varepsilon}\right)\right]_{k}^{\ell}\left[e_{W}\left(t_{\varepsilon}\right)\right]_{j}^{\ell^{\prime}}\left(\partial_{A_{\ell}} A_{\ell^{\prime}}-\sum_{i} \Gamma_{\ell, \ell^{\prime}}^{i} A_{i}\right)\left(\xi_{W^{\varepsilon}}^{\sharp}\left(t_{\varepsilon}\right)\right)\right\}\left(\Delta\left(W_{k}\right) \Delta\left(W_{j}\right)-\varepsilon \eta_{k}^{j}\right) .
\end{aligned}
$$

Lemma 5.1. The process $\xi_{W}^{\sharp}$ has the same law as the process $Z_{W^{\varepsilon}}$ defined in (3).

Proof. By Proposition 4.2, $\widehat{W}_{\ell}(t)-\widehat{W}_{\ell}\left(t_{\varepsilon}\right):=\sum_{k}\left[e_{W}\left(t_{\varepsilon}\right)\right]_{k}^{\ell}\left(W_{k}(t)-W_{k}\left(t_{\varepsilon}\right)\right)$ are the increments of an $\mathbb{R}^{d}$-valued Brownian motion $\widehat{W}$; we get

$$
\begin{aligned}
& \xi_{W}^{\sharp}(t)-\xi_{W}^{\sharp}\left(t_{\varepsilon}\right)=A_{0}\left(\xi_{W}^{\sharp}\left(t_{\varepsilon}\right)\right)\left(t-t_{\varepsilon}\right)+\sum_{k} A_{k}\left(\xi_{W}^{\sharp}\left(t_{\varepsilon}\right)\right)\left(\widehat{W}_{k}(t)-\widehat{W}_{k}\left(t_{\varepsilon}\right)\right) \\
& \quad+\frac{1}{2} \sum_{k, s}\left(\partial_{A_{k}} A_{s}-\sum_{i} \Gamma_{k, s}^{i} A_{i}\right)\left(\xi_{W}^{\sharp}\left(t_{\varepsilon}\right)\right)\left(\left(\widehat{W}_{k}(t)-\widehat{W}_{k}\left(t_{\varepsilon}\right)\right)\left(\widehat{W}_{s}(t)-\widehat{W}_{s}\left(t_{\varepsilon}\right)\right)-\varepsilon \eta_{k}^{s}\right) .
\end{aligned}
$$


By Eq. (6), we have $2 \sum_{i} \Gamma_{k, s}^{i} A_{i}=\left[A_{k}, A_{s}\right]+\sum_{i}\left(\left\langle\left[A_{i}, A_{s}\right], A_{k}\right\rangle A_{i}+\left\langle\left[A_{i}, A_{k}\right], A_{s}\right\rangle A_{i}\right)$, where the first term is antisymmetric in $k, s$ and does not contribute; the remaining sum is symmetric in $k, s$. Thus we get $-\sum_{i, k, s} \Gamma_{k, s}^{i} A_{i} \Delta\left(\widehat{W}_{k}\right) \Delta\left(\widehat{W}_{s}\right)=\sum_{i, k, s} A_{i}\left\langle\left[A_{s}, A_{i}\right], A_{k}\right\rangle \Delta\left(\widehat{W}_{k}\right) \Delta\left(\widehat{W}_{s}\right)$ which proves Lemma 5.1.

Lemma 5.2. We have $\mathbb{E}\left[\sup _{t \in[0,1]}\left\|\xi_{W}^{\sharp}(t)-\tilde{\xi}_{W^{\varepsilon}}(t)\right\|_{\mathbb{R}^{d}}^{2}\right] \leqslant c \varepsilon^{2}$.

Proof. The following method of introducing a parameter $\lambda$ and differentiating with respect to $\lambda$, is continuously used in [4]. For $\lambda \in[0,1]$, let $e^{\lambda}:=\lambda e_{W}+(1-\lambda) e_{W^{\varepsilon}}$ and define the process $\xi_{W}^{\lambda}$ by

$$
\begin{aligned}
& \xi_{W}^{\lambda}(t)-\xi_{W}^{\lambda}\left(t_{\varepsilon}\right)=A_{0}\left(\xi_{W}^{\lambda}\left(t_{\varepsilon}\right)\right)\left(t-t_{\varepsilon}\right)+\sum_{k, \ell}\left[e_{W}^{\lambda}\left(t_{\varepsilon}\right)\right]_{k}^{\ell} A_{\ell}\left(\xi_{W}^{\lambda}\left(t_{\varepsilon}\right)\right) \Delta\left(W_{k}\right) \\
& \quad+\frac{1}{2} \sum_{k, j}\left\{\sum_{\ell, \ell^{\prime}}\left[e_{W}^{\lambda}\left(t_{\varepsilon}\right)\right]_{k}^{\ell}\left[e_{W}^{\lambda}\left(t_{\varepsilon}\right)\right]_{j}^{\ell^{\prime}}\left(\partial_{A_{\ell}} A_{\ell^{\prime}}-\sum_{i} \Gamma_{\ell, \ell^{\prime}}^{i} A_{i}\right)\left(\xi_{W^{\varepsilon}}^{\lambda}\left(t_{\varepsilon}\right)\right)\right\}\left(\Delta\left(W_{k}\right) \Delta\left(W_{j}\right)-\left(t-t_{\varepsilon}\right) \eta_{k}^{j}\right) .
\end{aligned}
$$

Let $u_{W}^{\lambda}:=\partial \xi / \partial \lambda$; then $\xi_{W}^{\sharp}(t)-\tilde{\xi}_{W^{\varepsilon}}(t)=\int_{0}^{1} u_{W}^{\lambda}(t) \mathrm{d} \lambda$. Denote by $A_{k}^{\prime},\left(\partial_{A_{\ell}} A_{\ell^{\prime}}\right)^{\prime},\left(\Gamma_{\ell, \ell^{\prime}}^{i} A_{i}\right)^{\prime}$ the matrices obtained by differentiating $A_{k}, \partial_{A_{\ell}} A_{\ell^{\prime}}, \Gamma_{\ell, \ell^{\prime}}^{i} A_{i}$ with respect to $\xi$, and consider the delayed matrix SDE

$$
\begin{aligned}
d J_{t \leftarrow t_{0}}= & {\left[A_{0}^{\prime}\left(\xi_{W}^{\lambda}\left(t_{\varepsilon}\right)\right) \mathrm{d} t+\sum_{k, \ell}\left[e_{W}^{\lambda}\left(t_{\varepsilon}\right)\right]_{k}^{\ell} A_{\ell}^{\prime}\left(\xi_{W}^{\lambda}\left(t_{\varepsilon}\right)\right) \mathrm{d} W_{k}+\frac{1}{2} \sum_{k, j}\left\{\sum_{\ell, \ell^{\prime}}\left[e_{W}^{\lambda}\left(t_{\varepsilon}\right)\right]_{k}^{\ell}\left[e_{W}^{\lambda}\left(t_{\varepsilon}\right)\right]_{j}^{\ell^{\prime}}\right.\right.} \\
& \left.\left.\times\left(\left(\partial_{A_{\ell}} A_{\ell^{\prime}}\right)^{\prime}-\sum_{i}\left(\Gamma_{\ell, \ell^{\prime}}^{i} A_{i}\right)^{\prime}\right)\left(\xi_{W^{\varepsilon}}^{\lambda}\left(t_{\varepsilon}\right)\right)\right\}\left(\Delta\left(W_{k}\right) \mathrm{d} W_{j}(t)+\Delta\left(W_{j}\right) \mathrm{d} W_{k}(t)-\eta_{k}^{j} \mathrm{~d} t\right)\right] J_{t \leftarrow t_{0}}
\end{aligned}
$$

with initial condition $J_{t_{0} \leftarrow t_{0}}=\mathrm{Id}$. Then

$$
\begin{aligned}
u_{W}^{\lambda}(T)= & J_{T \leftarrow t_{0}} \int_{0}^{T} J_{t \leftarrow t_{0}}^{-1}\left[\sum_{k, \ell}\left[e_{W}\left(t_{\varepsilon}\right)-e_{W^{\varepsilon}}\left(t_{\varepsilon}\right)\right]_{k}^{\ell} A_{\ell}\left(\xi_{W}^{\lambda}\left(t_{\varepsilon}\right)\right) \mathrm{d} W_{k}(t)+\sum_{k, j}\left\{\sum_{\ell, \ell^{\prime}}\left[e_{W}\left(t_{\varepsilon}\right)-e_{W^{\varepsilon}}\left(t_{\varepsilon}\right)\right]_{k}^{\ell}\right.\right. \\
& \left.\left.\times\left[e_{W}^{\lambda}\left(t_{\varepsilon}\right)\right]_{j}^{\ell^{\prime}}\left(\partial_{A_{\ell}} A_{\ell^{\prime}}-\sum_{i} \Gamma_{\ell, \ell^{\prime}}^{i} A_{i}\right)\left(\xi_{W^{\varepsilon}}^{\lambda}\left(t_{\varepsilon}\right)\right)\right\}\left(\Delta\left(W_{k}\right) \mathrm{d} W_{j}(t)+\Delta\left(W_{j}\right) \mathrm{d} W_{k}(t)-\eta_{k}^{j} \mathrm{~d} t\right)\right]
\end{aligned}
$$

which along with (12) proves Lemma 5.2.

\section{References}

[1] A.-B. Cruzeiro, P. Malliavin, Renormalized differential geometry on path space: structural equation, curvature, J. Funct. Anal. 139 (1996) 119-181.

[2] P.E. Kloeden, E. Platen, Numerical Solution of Stochastic Differential Equations, Springer-Verlag, Berlin, 1992.

[3] P. Malliavin, Paramétrix trajectorielle pour un opérateur hypoelliptique et repère mobile stochastique, C. R. Acad. Sci. Paris, Sér. A-B 281 (1975) A241-A244.

[4] P. Malliavin, A. Thalmaier, Numerical error for SDE: asymptotic expansion and hyperdistributions, C.R. Math. Acad. Sci. Paris, Sér. I 336 (2003) 851-856.

[5] F. Malrieu, Convergence to equilibrium for granular media equations and their Euler schemes, Ann. Appl. Probab. 13 (2003) $540-560$.

[6] G.N. Milstein, Numerical Integration of Stochastic Differential Equations, in: Math. Appl., vol. 313, Kluwer Academic, Dordrecht, 1995. Translated and revised from the 1988 Russian original.

[7] D.W. Stroock, S.R.S. Varadhan, Multidimensional Diffusion Processes, in: Grundlehren Math. Wiss., vol. 233, Springer-Verlag, Berlin, 1979.

[8] C. Villani, Topics in Optimal Transportation, in: Grad. Stud. Math., vol. 58, Americal Mathematical Society, Providence, RI, 2003. 